\title{
EUPHORBIO VALDEVILLOSOCARPAE-INULETUM SALICINAE ASS. NOVA PÎNZARU, CANTEMIR \& JARDAN (TRIFOLION MEDII T. MÜLLER 1962) IN THE REPUBLIC OF MOLDOVA
}

\author{
$\underline{\text { Pavel PINZZARU }}^{1 *}$, Valentina CANTEMIR ${ }^{1}$, Natalia JARDAN ${ }^{2}$ \\ 1 "Alexandru Ciubotaru" National Botanical Garden (Institute), Chişinău - Republic of Moldova \\ 2 “Codru” Scientific Reserve, Lozova comune, Străşeni district - Republic of Moldova \\ ${ }^{*}$ Corresponding author. E-mail: p_panzaru@yahoo.it
}

\begin{abstract}
The vegetation of the "Peacock" glades in the "Codru” Scientific Reserve, Republic of Moldova, based on 15 relevés has been grouped in a new association Euphorbio valdevillosocarpae-Inuletum salicinae Pînzaru, Cantemir et Jardan, ass. nova, h.l., alliance Trifolion medii T. Müller 1962, ord. Origanetalia vulgaris T. Müller 1962, cl. TRIFOLIO-GERANIETEA SANGUINEI T. Müller 1962. The association consists of mesophilic phytocenoses, formed on slightly acidic, typical gray soils, at an altitude of 330$336 \mathrm{~m}$. Hemicryptophytes predominate in the phytocoenoses of this association $(74.1 \%)$, among the more numerous floristic elements, there are the Eurasian ones (53.7\%), followed by the European ones (16.6\%) and the Central European ones (6.4\%).
\end{abstract}

Keywords: characteristic species, ecology, Euphorbio valdevillosocarpae-Inuletum salicinae ass. nova, range, Republic of Moldova.

\section{Introduction}

The vegetation of glades in the Republic of Moldova, for the most part, was studied from a phytosociological point of view, without identifying the plant associations, except for the glades in the arid cliff forests, which consist of phytocoenoses grouped in the association Inulo ensifoliae-Anthericetum ramosi Pînzaru et Coldea 2006 em. Pînzaru 2016, 2017. This article describes a new association - Euphorbio valdevillosocarpae-Inuletum salicinae, from the "Peacock" glades of the "Codru" Scientific Reserve. The "Peacock" glades are located on high hills, in the plots 43 and 52, surrounded by sessile oak forests (Quercus petraea) near Stejăreni village, Străşeni district.

Inula salicina L. (Figure 1) is a hemicryptophyte, Eurasian species, (xeromesophilic-) mesophilic (-mesohygrophilic), occurs in riverside meadows, glades and forest clearings, from hilly to mountainous areas, being part of the floristic composition of various associations. The association Agropyro elongatae-Inuletum salicinae Şerbănescu 1965 (Al. Plantagini salsae-Artemision santonicae Sheleag-Sosonko et Solomakha in Lysenko, Mucina et Iakushenko 2011) [DUBYNA \& al. 2019] is found in the meadow vegetation of Ukraine, and the association Violo elatioris-Inuletum salicinae Didier et Royer 1989 (Al. Molinion caeruleae Koch 1926) [BENSETTITI \& al. 2005] occurs in the hilly meadows of France. In the vegetation of Romania, there are no associations of Inula salicina, but it occurs as an accompanying species in other associations [COLDEA \& al. 2012; CHIFU \& al. 2014].

The characteristic species Euphorbia valdevillosocarpa Arvat et Nyár. [=E. volhynica auct. mold. non Besser ex Racib.] (Figure 2) is a Central European geoelement (endemic), occurring in Romania, the Republic of Moldova and Ukraine (western part). It is

Received: 5 October 2020 / Revised: 22 November 2020 / Accepted: 28 November 2020 
a mesophile and grows in glades, forest edges and sparse forests on hilly terrain [GELTMAN, 1996; SÂRBU \& al. 2013]. In the Republic of Moldova, it is rarely found, it has been observed that it is somewhat more common (abundance + coverage from + to 2 and constancy $\mathrm{V})$ in the phytocenoses of Inula salicina in the "Peacock" glades.

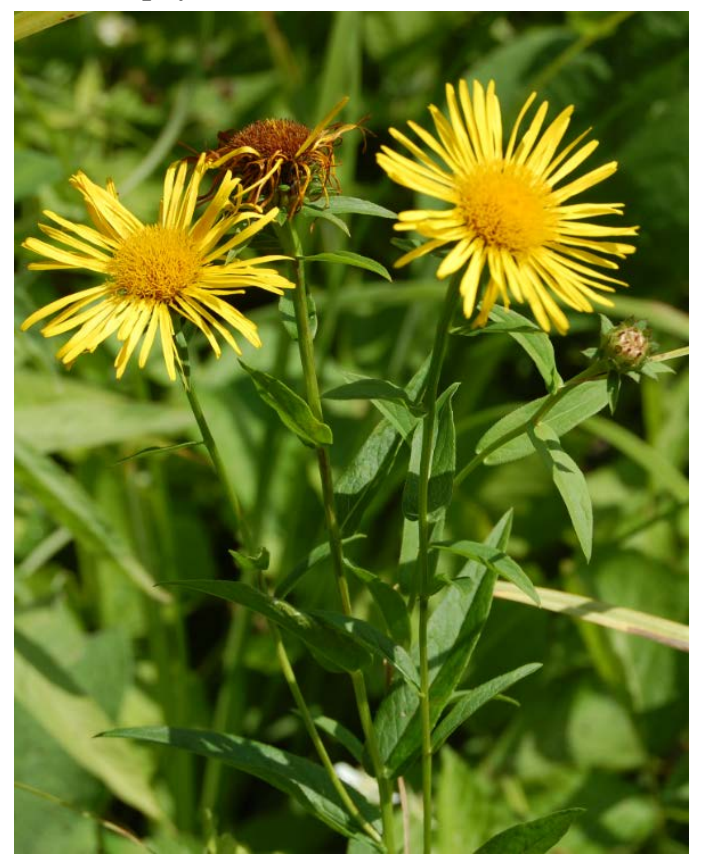

Figure 1. Inula salicina L.

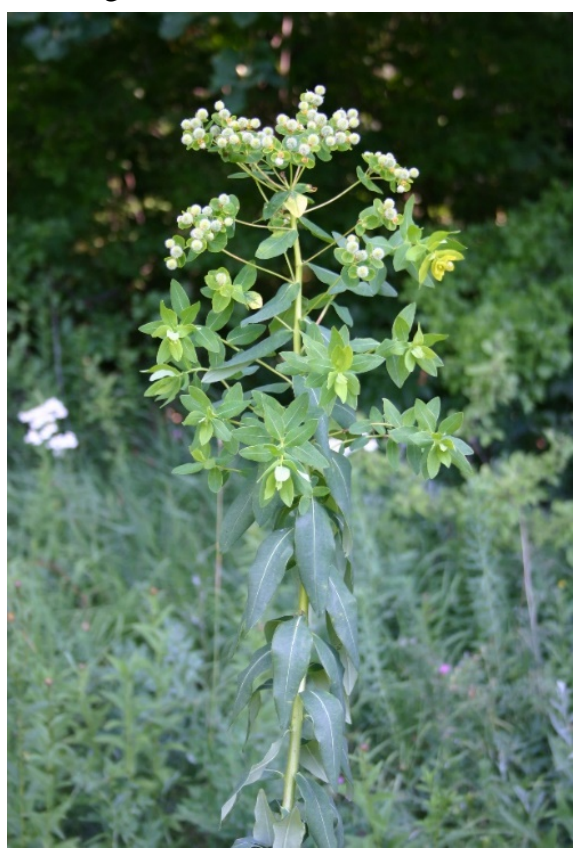

Figure 2. Euphorbia valdevillosocarpa Arvat et Nyár.

\section{Materials and methods}

The phytosociological research was conducted in June-September, 2020, according to the Braun-Blanquet approach [BRAUN-BLANQUET, 1964]. The area of the relevés was $100 \mathrm{~m}^{2}$ [CRISTEA \& al. 2004]. Species nomenclature followed PÎNZARU \& SÎRBU, 2016. The average annual temperature and precipitation were indicated according to the Atlas of Climate Resources of the Republic of Moldova [NEDEALCOV \& al. 2013]. The soils according to the monograph "The Soils of Moldova” [URSU, 2011].

\section{Results and discussions}

The plant communities of Inula salicina L. with Euporbia valdevillosocarpa Arvat et Nyár. and other species, in the "Peacock" glades, occur on slightly humic and slightly acidic typical gray soil, at an altitude of 330-336 m. The height of the hills and the slightly acidic soil create favourable conditions for the development of species characteristic of the class MOLINIO-ARRHENATHERETEA Tx. 1937, such as: Briza media, Hypochaeris maculata, Ornithogalum pyrenaicum, Serratula coronata, Serratula tinctoria, Silene atropurpurea etc. 
Pavel PÎNZARU \& al.

These phytocoenoses have a compact coverage (100\%), and a yellowish color predominates in the landscape during the flowering period of the dominant species.

\section{Ass. Euphorbio valdevillosocarpae-Inuletum salicinae}

Pînzaru, Cantemir et Jardan, ass. nova, hoc loco

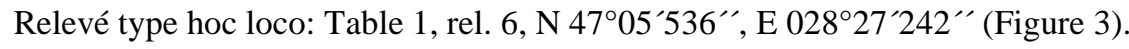

Synoptic table hoc loco: Table 1, 15 relevés

The total area of the phytocoenoses of the association described in this article comprises about 2.6 ha.

Locations: Altitude: 330-336 m. Relief: Central Moldavian Plateau, on top of flat or slightly sloping hills $\left(5^{\circ}\right)$, with southern exposure. Soil: typical gray, slightly humic, slightly acidic, formed on loamy-clayey rocks. Climate: temperate-continental, the average annual temperature is $10.0-10.5^{\circ} \mathrm{C}$, and the average annual precipitation varies between 650 and 700 $\mathrm{mm}$.

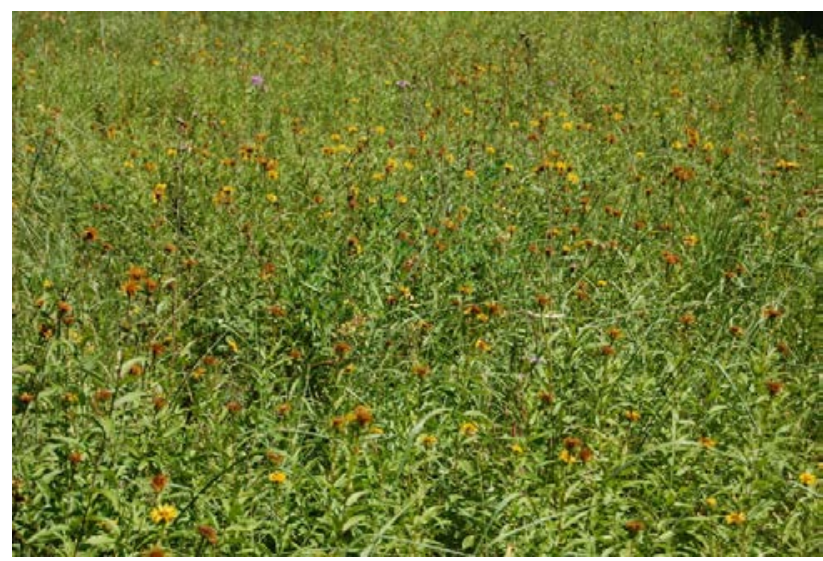

Figure 3. As. Euphorbio valdevillosocarpae-Inuletum salicinae ass. nova (type) - 21 July 2020, Stejăreni village, Străşeni district.

Characteristic species: Inula salicina, Euphorbia valdevillosocarpa.

Constant species: Centaurea jacea, Galium verum, Peucedanum cervaria, Tanacetum corymbosum, Serratula tinctoria, Achillea pannonica, Iris graminea, Filipendula vulgaris, Stachys officinalis, Briza media.

Rare species: Ornithogalum pyrenaicum (= O. flavescens Lam.) [Endangered (EN)], included in the Red Book of Moldova (2015), Serratula coronata [Endangered (EN), included in the Red Book of Moldova (2015), Silene atropurpurea (= Viscaria atropurpurea Griseb.) [Critically Endangered (CR)], included in the Red Book of Moldova (2015), Asparagus tenuifolius (Least Consern (LC)] (Legea... 1998), Briza media [Nearly Threatened (NT)] (Legea...1998), Doronicum hungaricum [Vulnerable (VU)], (Legea...1998), Iris variegata [Vulnerable (VU)] (Legea...1998), Luzula campestris [Nearly Threatened (NT)] (Legea...1998), Orchis mascula [Critically Endangered (CR)] (Legea...1998), Hypochaeris maculata L. [Vulnerable (VU)] (Legea...1998).

Structure: The overall vegetation cover is $100 \%$ (Figure 3). Although the plants in these phytocenoses are of different heights, from creeping to erect plants - about $150 \mathrm{~cm}$ tall, only the dominant species Inula salicina and Euphorbia valdevillosocarpa form a welldefined layer, reaching a height of $70-90 \mathrm{~cm}$, the other species have an insignificant 
abundance. The species of small plants (up to $\pm 15 \mathrm{~cm}$ tall): Viola odorata, Lysimachia nummularia, Melampyrum cristatum, Luzula campestris, Glechoma hirsuta, Fragaria viridis, Primula veris, Prunella vulgaris etc., in some places, they have a cover between 5$10 \%$, and the tall species (120-150 cm) have sporadic distribution: Peucedanum cervaria, $P$. alsaticum, Thalictrum lucidum, Serratula coronata, Cirsium pannonicum.

Floristic composition. In the 15 studied relevés, 108 species of vascular plants have been identified, and 47 of them are characteristic of coenotaxa of the class TRIFOLIOGERANIETEA SANGUINEI T. Müller 1962, 21 species - cl. MOLINIOARRHENATHERETEA Tx. 1937, 11 species - cl. QUERCO-FAGETEA Br.-Bl. et Vlieger in Vlieger 1937, 3 species - cl. CRATAEGO-PRUNETEA Tx. 1962, and 26 species - Variae syntaxa.

The spectrum of life forms includes: hemicryptophytes $(\mathrm{H})=80$ species $(74.1 \%)$,

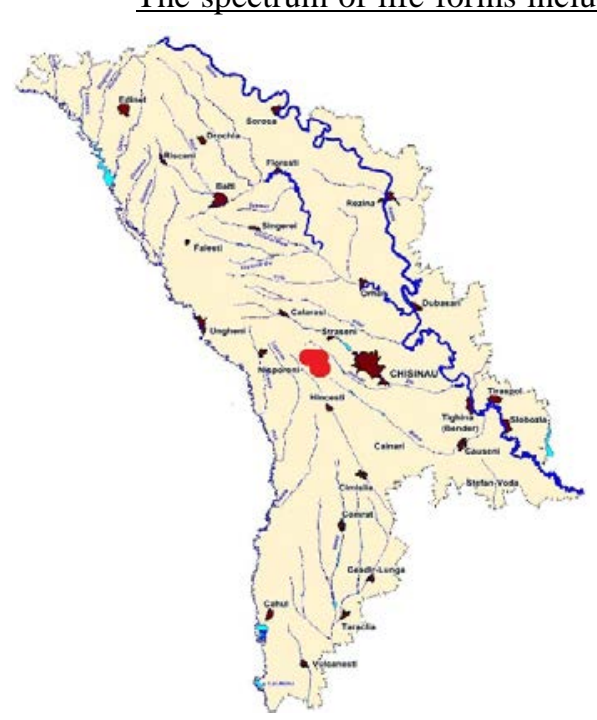

Figure 4. Locations of the ass. Euphorbio geophytes $(G)=10$ species $(\approx 9.3 \%)$, chamaephytes $(\mathrm{Ch})=4$ species $(3.7 \%)$, nanophanerophytes $(\mathrm{Phn})=4$ species $(3.7 \%)$, therophytes $(\mathrm{Th})=8$ species $(7.4 \%)$, hemitherophytes $(\mathrm{TH})=2$ species $(1.8 \%)$.

In the spectrum of geoelements, the Eurasian ones predominate (Eua) $=58$ species $(53.7 \%)$, followed by the European ones (Eur) $=18$ species (16.6\%) and Central European (Euc) $=7$ species (6.4\%), other geoelements are represented by 1 to 4 species.

According to the soil humidity indices, in the phytocoenoses of the given association, there are 56 mesophilic (ms) species (51.7\%) and 52 xeromesophilic (xm) species (48.3\%), for these reasons, we have included this association in the alliance Trifolion medii T. Müller 1962, order Origanetalia vulgaris T. Muller 1962.

Range (Figure 4). The phytocoenoses of the association Euphorbio valdevillocarpaeInuletum salicinae occur in the glades of sessile oak forests (plots no. 43 and 52), near Stejăreni village, Străşeni district.

Territorial protection. The phytocoenoses of the above-mentioned association are protected on the territory of the "Codru" Scientific Reserve.

Conservation value. The plant communities of the highlighted association are of high conservation value; they are rare and include 10 rare, protected species, among them, there are 3 species that are listed in the Red Book of the Republic of Moldova (2015). 
Pavel PÎNZARU \& al.

\begin{tabular}{|c|c|c|c|c|c|c|c|c|c|c|c|c|c|c|c|c|c|c|c|}
\hline \multirow{7}{*}{ E } & \multirow{7}{*}{ 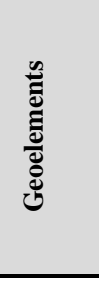 } & \multirow{7}{*}{ 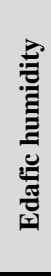 } & Relevé no. & 1 & 2 & 3 & 4 & 5 & $* 6$ & 7 & 8 & 9 & 10 & 11 & 12 & 13 & 14 & 15 & \multirow[t]{7}{*}{$\mathbf{K}$} \\
\hline & & & Altitude (m) & 330 & 331 & 331 & 335 & 335 & 335 & 335 & 335 & 335 & 335 & 335 & 335 & 336 & 336 & 336 & \\
\hline & & & Aspect & $\mathrm{S}$ & $\mathrm{S}$ & $\mathrm{S}$ & - & - & - & - & - & - & - & - & - & - & - & - & \\
\hline & & & Slope $\left(^{\circ}\right)$ & 5 & 5 & 5 & - & - & - & - & - & - & - & - & - & - & - & - & \\
\hline & & & General coverage $(\%)$ & 100 & 100 & 100 & 100 & 100 & 100 & 100 & 90 & 100 & 100 & 100 & 100 & 100 & 100 & 100 & \\
\hline & & & Surface of relevé & 100 & 100 & 100 & 100 & 100 & 100 & 100 & 100 & 100 & 100 & 100 & 100 & 100 & 100 & 100 & \\
\hline & & & Number of species & 30 & 24 & 39 & 36 & 26 & 36 & 25 & 43 & 29 & 41 & 29 & 24 & 32 & 31 & 34 & \\
\hline & & & $\underline{\text { Charact. species }}$ & & & & & & & & & & & & & & & & \\
\hline $\mathrm{H}$ & Eua & $\mathrm{ms}$ & Inula salicina & 4 & 4 & 4 & 4 & 4 & 4 & 3 & 4 & 4 & 3 & 4 & 4 & 4 & 4 & 3 & V \\
\hline $\mathrm{H}$ & Euc & $\mathrm{ms}$ & $\begin{array}{l}\text { Euphorbia valdevillosocarpa } \\
\text { Trifolion medii }\end{array}$ & 1 & 1 & 1 & 1 & 1 & 2 & 2 & 1 & 1 & 2 & 1 & 1 & 1 & 1 & 1 & V \\
\hline $\mathrm{H}$ & Eua & $\mathrm{ms}$ & Centaurea jacea & + & 1 & 1 & + & 1 & + & + & 1 & 1 & - & 1 & + & 1 & - & + & $\mathrm{V}$ \\
\hline $\mathrm{H}$ & Eur & $\mathrm{ms}$ & Achillea pannonica & 1 & + & + & 1 & $\mathrm{r}$ & + & $\mathrm{r}$ & 1 & + & 1 & + & - & - & + & - & IV \\
\hline $\mathrm{H}$ & Eua & $\mathrm{ms}$ & Lathyrus pratensis & + & + & - & + & + & + & - & - & + & - & - & + & + & + & - & III \\
\hline $\mathrm{H}$ & Eur & $\mathrm{ms}$ & Knautia arvensis & - & - & - & - & - & - & - & - & - & $\mathrm{r}$ & - & - & - & - & - & I \\
\hline $\mathrm{H}$ & Eua & $\mathrm{ms}$ & Leucanthemum vulgare & - & - & - & - & - & - & - & $\mathrm{r}$ & - & $\mathrm{r}$ & - & - & - & - & $\mathrm{r}$ & I \\
\hline $\mathrm{H}$ & Eua & $\mathrm{ms}$ & $\begin{array}{l}\text { Trifolium medium } \\
\text { Origanetalia vulgaris }\end{array}$ & - & - & - & - & - & - & - & - & - & - & - & - & - & - & $\mathrm{r}$ & I \\
\hline $\mathrm{H}$ & Eua & $\mathrm{ms}$ & Galium verum & + & + & + & + & + & + & $\mathrm{r}$ & + & + & + & + & + & $\mathrm{r}$ & + & 2 & V \\
\hline $\mathrm{H}$ & Eua & $\mathrm{ms}$ & Filipendula vulgaris & $\mathrm{r}$ & + & + & + & - & $\mathrm{r}$ & $\mathrm{r}$ & + & - & 1 & $\mathrm{r}$ & - & + & + & $\mathrm{r}$ & IV \\
\hline $\mathrm{H}$ & Eua & $\mathrm{ms}$ & Galatella sedifolia & + & + & - & - & - & 1 & 1 & - & - & - & 1 & 1 & 1 & 1 & + & III \\
\hline $\mathrm{H}$ & Eur & $\mathrm{xm}$ & Trifolium alpestre & - & - & - & + & + & + & $\mathrm{r}$ & + & - & $\mathrm{r}$ & - & - & $\mathrm{r}$ & + & + & III \\
\hline $\mathrm{H}$ & Euc & $\mathrm{xm}$ & Valeriana collina & $\mathrm{r}$ & - & $\mathrm{r}$ & - & - & - & - & - & - & - & $\mathrm{r}$ & $\mathrm{r}$ & $\mathrm{r}$ & - & $\mathrm{r}$ & II \\
\hline $\mathrm{H}$ & Eur & $\mathrm{ms}$ & Lathyrus sylvestris & - & - & - & - & - & $\mathrm{r}$ & - & - & - & - & - & - & - & - & - & I \\
\hline $\mathrm{H}$ & Eua & $\mathrm{xm}$ & Medicago falcata & - & - & - & $\mathrm{r}$ & - & $\mathrm{r}$ & - & - & - & $r$ & - & - & - & - & - & I \\
\hline $\mathrm{H}$ & Eua & $\mathrm{xm}$ & Origanum vulgare & - & - & - & - & $\mathrm{r}$ & - & - & - & - & $\mathrm{r}$ & - & - & - & - & - & I \\
\hline $\mathrm{H}$ & Eua & $\mathrm{ms}$ & Primula veris & - & - & $\mathrm{r}$ & - & - & + & - & - & - & - & - & - & - & - & - & I \\
\hline $\mathrm{H}$ & Euc-M & $\mathrm{xm}$ & Securigera varia & - & - & - & - & + & - & - & - & - & $\mathrm{r}$ & - & - & $\mathrm{r}$ & - & - & I \\
\hline
\end{tabular}


EUPHORBIO VALDEVILLOSOCARPAE-INULETUM SALICINAE ASS. NOVA PÎNZARU ...

\begin{tabular}{|c|c|c|c|c|c|c|c|c|c|c|c|c|c|c|c|c|c|c|c|}
\hline $\mathrm{H}$ & Eua & $\mathrm{ms}$ & Silene vulgaris & - & - & $\mathrm{r}$ & - & - & - & - & $\mathrm{r}$ & - & - & - & - & - & - & - & I \\
\hline $\mathrm{H}$ & Circ & $\mathrm{xm}$ & Solidago virgaurea & - & - & - & r & - & - & - & - & - & - & - & - & - & - & - & I \\
\hline Th & Eua & $\mathrm{ms}$ & $\begin{array}{l}\text { Vicia hirsuta } \\
\text { Geranion sanguinei }\end{array}$ & - & - & - & - & - & - & $\mathrm{r}$ & - & - & - & - & $\mathrm{r}$ & - & - & - & I \\
\hline $\mathrm{H}$ & Eur & $\mathrm{xm}$ & Peucedanum cervaria & $r$ & 1 & + & + & + & + & - & + & + & $\mathrm{r}$ & $\mathrm{r}$ & $\mathrm{r}$ & - & $\mathrm{r}$ & 1 & V \\
\hline $\mathrm{H}$ & Euc & $\mathrm{xm}$ & Galium rubioides & - & + & - & - & - & - & - & - & $\mathrm{r}$ & $\mathrm{r}$ & - & - & $\mathrm{r}$ & $\mathrm{r}$ & $\mathrm{r}$ & II \\
\hline Th & Eur & $\mathrm{xm}$ & Melampyrum cristatum & + & - & - & $\mathrm{r}$ & $\mathrm{r}$ & $\mathrm{r}$ & $\mathrm{r}$ & + & - & - & - & - & - & $\mathrm{r}$ & - & II \\
\hline G & P-P-B & $\mathrm{xm}$ & Iris variegata & - & - & - & - & - & - & - & - & - & $\mathrm{r}$ & $\mathrm{r}$ & - & $\mathrm{r}$ & - & - & I \\
\hline $\mathrm{H}$ & Euc & $\mathrm{xm}$ & Peucedanum alsaticum & - & - & - & - & r & - & - & - & - & $\mathrm{r}$ & - & - & - & - & - & I \\
\hline $\mathrm{H}$ & Euc-M & $\mathrm{xm}$ & Prunella laciniata & - & - & - & - & - & - & - & - & - & - & - & - & - & + & - & I \\
\hline $\mathrm{H}$ & Med & $\mathrm{xm}$ & Silene coronaria & - & - & - & - & - & - & - & - & $\mathrm{r}$ & - & - & - & - & - & - & I \\
\hline $\mathrm{H}$ & Eua & $\mathrm{xm}$ & Trifolium montanum & - & - & - & - & - & - & - & + & - & - & + & - & - & - & - & I \\
\hline $\mathrm{H}$ & Eua & $\mathrm{xm}$ & $\begin{array}{l}\text { Veronica spicata } \\
\text { Antherico ramosi- } \\
\text { Geranietalia sanguinei }\end{array}$ & - & - & - & $\mathrm{r}$ & - & - & - & - & - & - & - & - & - & $\mathrm{r}$ & - & I \\
\hline $\mathrm{H}$ & Eua & $\mathrm{xm}$ & Tanacetum corymbosum & $\mathrm{r}$ & $\mathrm{r}$ & $\mathrm{r}$ & $\mathrm{r}$ & $\mathrm{r}$ & $\mathrm{r}$ & $\mathrm{r}$ & + & $\mathrm{r}$ & $\mathrm{r}$ & - & $\mathrm{r}$ & + & - & + & $\mathrm{V}$ \\
\hline G & Pont-M & $\mathrm{xm}$ & Iris graminea & $\mathrm{r}$ & $r$ & $\mathrm{r}$ & $\mathrm{r}$ & $\mathrm{r}$ & $\mathrm{r}$ & $\mathrm{r}$ & - & r & $\mathrm{r}$ & - & - & $\mathrm{r}$ & $\mathrm{r}$ & - & IV \\
\hline $\mathrm{H}$ & P-P & $\mathrm{xm}$ & Cirsium pannonicum & - & - & - & - & - & - & - & + & $\mathrm{r}$ & $\mathrm{r}$ & - & - & $\mathrm{r}$ & $\mathrm{r}$ & + & II \\
\hline $\mathrm{TH}$ & Med & $\mathrm{xm}$ & Arabis sagittata & - & - & - & - & - & - & - & - & - & $\mathrm{r}$ & - & - & $\mathrm{r}$ & - & $\mathrm{r}$ & I \\
\hline $\mathrm{H}$ & Eua & $\mathrm{xm}$ & $\begin{array}{l}\text { Nepeta nuda } \\
\text { Trifolio-Geranietea }\end{array}$ & - & - & $\mathrm{r}$ & - & - & - & - & $\mathrm{r}$ & - & - & - & - & - & - & - & I \\
\hline $\mathrm{H}$ & Eua & $\mathrm{ms}$ & Stachys officinalis & + & - & $\mathrm{r}$ & $\mathrm{r}$ & $\mathrm{r}$ & - & $\mathrm{r}$ & $\mathrm{r}$ & - & $\mathrm{r}$ & $\mathrm{r}$ & $\mathrm{r}$ & $\mathrm{r}$ & $\mathrm{r}$ & $\mathrm{r}$ & IV \\
\hline $\mathrm{H}$ & Circ & $\mathrm{xm}$ & Clinopodium vulgare & $\mathrm{r}$ & - & $\mathrm{r}$ & $\mathrm{r}$ & - & + & - & + & - & + & $\mathrm{r}$ & - & - & $\mathrm{r}$ & - & III \\
\hline $\mathrm{H}$ & Pont & $\mathrm{xm}$ & Dianthus membranaceus & - & - & - & $\mathrm{r}$ & $\mathrm{r}$ & $\mathrm{r}$ & - & $\mathrm{r}$ & $\mathrm{r}$ & - & $\mathrm{r}$ & - & - & $\mathrm{r}$ & $\mathrm{r}$ & III \\
\hline $\mathrm{H}$ & Eua & $\mathrm{xm}$ & $\begin{array}{l}\text { Veronica teucrium } \\
\text { Campanula glomerata }\end{array}$ & $\mathrm{r}$ & + & $\mathrm{r}$ & $\mathrm{r}$ & $\mathrm{r}$ & $\mathrm{r}$ & $\mathrm{r}$ & $\mathrm{r}$ & $\mathrm{r}$ & - & - & - & - & - & - & III \\
\hline $\mathrm{H}$ & Eua & $\mathrm{xm}$ & var. cervicarioides & - & - & - & - & - & - & - & - & $\mathrm{r}$ & $\mathrm{r}$ & - & - & $\mathrm{r}$ & $\mathrm{r}$ & - & II \\
\hline $\mathrm{H}$ & Eua & $\mathrm{ms}$ & Hypericum perforatum & - & - & $\mathrm{r}$ & - & - & - & $\mathrm{r}$ & $\mathrm{r}$ & - & $\mathrm{r}$ & $\mathrm{r}$ & - & - & - & $\mathrm{r}$ & II \\
\hline $\mathrm{H}$ & Eua & $\mathrm{ms}$ & Vicia cracca & - & - & $\mathrm{r}$ & - & + & - & - & $\mathrm{r}$ & $\mathrm{r}$ & $\mathrm{r}$ & - & - & - & - & + & II \\
\hline $\mathrm{H}$ & Eua & $\mathrm{xm}$ & Campanula persicifolia & - & - & - & - & - & - & - & $\mathrm{r}$ & - & $\mathrm{r}$ & - & - & - & r & - & I \\
\hline
\end{tabular}


Pavel PÎNZARU \& al.

\begin{tabular}{|c|c|c|c|c|c|c|c|c|c|c|c|c|c|c|c|c|c|c|c|}
\hline & & & & & & & & & & & & & & & & & & & \\
\hline Th & Eur & $\mathrm{xm}$ & Dianthus armeria & - & - & - & - & - & - & - & - & - & - & - & $\mathrm{r}$ & - & - & - & I \\
\hline $\mathrm{H}$ & Eua & $\mathrm{ms}$ & Fragaria vesca & + & 1 & + & + & - & - & - & - & + & + & - & - & - & - & - & I \\
\hline $\mathrm{Ch}$ & Euc-M & $\mathrm{xm}$ & Teucrium chamaedrys & - & - & - & - & - & - & - & - & - & - & - & - & - & - & $\mathrm{r}$ & I \\
\hline $\mathrm{H}$ & Eur & $\mathrm{xm}$ & $\begin{array}{l}\text { Vincetoxicum hirundinaria } \\
\text { Molinio-. Arrhenatheretea s.l. }\end{array}$ & $\mathrm{r}$ & - & $\mathrm{r}$ & - & - & - & - & - & - & - & - & - & $\mathrm{r}$ & - & - & I \\
\hline $\mathrm{H}$ & Eua & $\mathrm{ms}$ & Serratula tinctoria & + & + & + & $\mathrm{r}$ & - & + & 1 & - & + & $\mathrm{r}$ & + & + & + & + & 1 & $\mathrm{~V}$ \\
\hline $\mathrm{H}$ & Eua & $\mathrm{ms}$ & Briza media & - & $\mathrm{r}$ & - & $\mathrm{r}$ & - & + & - & + & $\mathrm{r}$ & $\mathrm{r}$ & $\mathrm{r}$ & $\mathrm{r}$ & $\mathrm{r}$ & $\mathrm{r}$ & $\mathrm{r}$ & IV \\
\hline $\mathrm{H}$ & Eua & $\mathrm{ms}$ & Dactylis glomerata & + & + & + & - & $\mathrm{r}$ & - & 1 & - & - & - & - & + & + & - & - & III \\
\hline G & Euc-M & $\mathrm{ms}$ & Ornithogalum pyrenaicum & + & + & $\mathrm{r}$ & + & - & - & - & - & - & - & + & - & + & + & - & III \\
\hline $\mathrm{H}$ & Eur & $\mathrm{ms}$ & Salvia pratensis & $\mathrm{r}$ & $\mathrm{r}$ & $\mathrm{r}$ & $\mathrm{r}$ & $\mathrm{r}$ & - & - & $\mathrm{r}$ & $\mathrm{r}$ & - & - & - & - & - & - & III \\
\hline $\mathrm{H}$ & Eua & $\mathrm{ms}$ & Hypochaeris maculata & r & - & r & - & - & - & - & - & - & - & - & - & r & r & - & II \\
\hline $\mathrm{H}$ & Pont & $\mathrm{ms}$ & Serratula coronata & - & - & $\mathrm{r}$ & - & - & - & - & - & $\mathrm{r}$ & - & $\mathrm{r}$ & - & $\mathrm{r}$ & $\mathrm{r}$ & - & II \\
\hline $\mathrm{H}$ & Balc & $\mathrm{ms}$ & Silene atropurpurea & - & - & - & - & - & $\mathrm{r}$ & - & $\mathrm{r}$ & - & - & - & $\mathrm{r}$ & - & - & $\mathrm{r}$ & II \\
\hline $\mathrm{H}$ & Euc-Po & $\mathrm{ms}$ & Thalictrum lucidum & r & r & - & - & - & - & $\mathrm{r}$ & - & - & r & + & r & - & - & - & II \\
\hline $\mathrm{H}$ & Eua & $\mathrm{ms}$ & Calamagrostis epigejos & + & - & - & - & - & - & - & - & - & - & - & - & - & + & - & I \\
\hline $\mathrm{H}$ & Eua & $\mathrm{ms}$ & Leontodon hispidus & - & - & - & $\mathrm{r}$ & - & - & - & - & - & - & - & - & - & - & - & I \\
\hline $\mathrm{H}$ & Eua & $\mathrm{ms}$ & Lotus corniculatus & - & - & - & - & - & - & - & $\mathrm{r}$ & - & r & - & - & - & - & - & I \\
\hline $\mathrm{H}$ & Eur & $\mathrm{ms}$ & Luzula campestris & - & - & - & - & - & + & - & - & - & - & - & - & - & - & $\mathrm{r}$ & I \\
\hline $\mathrm{Ch}$ & Eur & $\mathrm{ms}$ & Lysimachia nummularia & $\mathrm{r}$ & - & + & + & - & - & - & - & - & - & - & - & - & - & - & I \\
\hline $\mathrm{H}$ & Eua & $\mathrm{ms}$ & Plantago media & - & - & - & - & $\mathrm{r}$ & - & - & r & $\mathrm{r}$ & - & - & - & - & - & - & I \\
\hline $\mathrm{H}$ & Circ & $\mathrm{ms}$ & Prunella vulgaris & - & - & - & - & - & - & - & - & - & - & - & - & - & - & $\mathrm{r}$ & I \\
\hline $\mathrm{H}$ & Circ & $\mathrm{ms}$ & Scutellaria galericulata & - & - & - & - & - & - & + & - & - & - & - & - & - & $\mathrm{r}$ & - & I \\
\hline $\mathrm{H}$ & Eua & $\mathrm{ms}$ & Stellaria graminea & - & - & - & r & - & - & - & $\mathrm{r}$ & - & - & - & - & - & - & $\mathrm{r}$ & I \\
\hline $\mathrm{H}$ & Eua & $\mathrm{ms}$ & Veronica longifolia & - & $\mathrm{r}$ & - & - & - & - & - & - & - & - & - & - & $\mathrm{r}$ & - & - & I \\
\hline $\mathrm{H}$ & Eua & $\mathrm{ms}$ & Viola jordanii & - & - & - & - & - & $\mathrm{r}$ & - & - & - & $\mathrm{r}$ & - & - & - & - & - & I \\
\hline $\mathrm{H}$ & Eua & $\mathrm{ms}$ & $\begin{array}{l}\text { Viola pumila } \\
\text { Crataego-Prunetea s.l. }\end{array}$ & - & - & - & - & - & - & - & - & - & - & - & - & + & - & - & I \\
\hline $\mathrm{Phn}$ & Eur & $\mathrm{xm}$ & Crataegus monogyna & $\mathrm{r}$ & - & - & $\mathrm{r}$ & - & $\mathrm{r}$ & - & $\mathrm{r}$ & $\mathrm{r}$ & $\mathrm{r}$ & $\mathrm{r}$ & + & - & - & - & III \\
\hline Phn & Eur & $\mathrm{xm}$ & Rosa canina & - & - & r & - & - & $\mathrm{r}$ & - & - & $\mathrm{r}$ & - & - & - & r & - & - & II \\
\hline
\end{tabular}


EUPHORBIO VALDEVILLOSOCARPAE-INULETUM SALICINAE ASS. NOVA PÎNZARU ...

\begin{tabular}{|c|c|c|c|c|c|c|c|c|c|c|c|c|c|c|c|c|c|c|c|}
\hline $\mathrm{Phn}$ & Eua & $\mathrm{xm}$ & $\begin{array}{l}\text { Prunus spinosa } \\
\text { Querco-Fagetea s.l. }\end{array}$ & - & - & - & - & - & - & - & + & - & $\mathrm{r}$ & - & - & - & - & - & I \\
\hline G & Pont & $\mathrm{xm}$ & Carex brevicollis & - & - & + & - & + & + & - & $\mathrm{r}$ & $\mathrm{r}$ & $\mathrm{r}$ & $\mathrm{r}$ & - & - & - & - & III \\
\hline G & Pont-M & $\mathrm{xm}$ & Asparagus tenuifolius & - & - & $\mathrm{r}$ & $\mathrm{r}$ & - & - & - & $\mathrm{r}$ & - & - & - & r & $\mathrm{r}$ & - & - & II \\
\hline $\mathrm{H}$ & Eua & $\mathrm{ms}$ & Ranunculus auricomus & - & - & $\mathrm{r}$ & $\mathrm{r}$ & $\mathrm{r}$ & - & - & $\mathrm{r}$ & - & - & $\mathrm{r}$ & r & - & - & - & II \\
\hline $\mathrm{H}$ & Med & $\mathrm{xm}$ & Viola odorata & $\mathrm{r}$ & - & $\mathrm{r}$ & + & - & $\mathrm{r}$ & - & $\mathrm{r}$ & - & - & - & - & - & - & - & II \\
\hline G & P-P-B & $\mathrm{ms}$ & Doronicum hungaricum & - & $\mathrm{r}$ & - & - & - & - & - & - & $\mathrm{r}$ & - & - & - & - & - & - & I \\
\hline $\mathrm{H}$ & Pont-M & $\mathrm{xm}$ & Glechoma hirsuta & - & - & + & - & - & - & + & - & - & + & - & - & - & - & - & I \\
\hline $\mathrm{H}$ & Eua & $\mathrm{ms}$ & Brachypodium sylvaticum & - & - & + & - & - & - & - & - & - & - & - & - & - & - & - & I \\
\hline $\mathrm{H}$ & Eua & $\mathrm{ms}$ & Hypericum hirsutum & - & - & $\mathrm{r}$ & - & - & - & - & - & - & - & - & - & - & - & - & I \\
\hline $\mathrm{H}$ & Euc & $\mathrm{xm}$ & Lathyrus niger & - & - & $\mathrm{r}$ & - & - & - & - & - & - & - & - & - & - & - & - & I \\
\hline G & Eur & $\mathrm{ms}$ & Orchis mascula & - & - & - & - & - & $\mathrm{r}$ & - & - & - & - & - & - & - & - & - & I \\
\hline $\mathrm{Phn}$ & Eur & $\mathrm{xm}$ & $\begin{array}{l}\text { Pyrus pyraster } \\
\text { Variae syntaxa }\end{array}$ & - & - & - & - & - & - & - & - & $\mathrm{r}$ & - & - & - & - & - & - & I \\
\hline $\mathrm{G}$ & Eua & $\mathrm{ms}$ & Elymus repens & 1 & 1 & 1 & + & - & 1 & - & 1 & 1 & 1 & 2 & 1 & 1 & 1 & - & IV \\
\hline $\mathrm{H}$ & Eua & $\mathrm{xm}$ & Festuca valesiaca & + & - & - & + & + & + & - & 1 & - & + & - & - & - & + & + & III \\
\hline $\mathrm{Ch}$ & Eua & $\mathrm{xm}$ & Artemisia austriaca & - & + & $\mathrm{r}$ & $\mathrm{r}$ & - & - & - & $\mathrm{r}$ & - & - & - & - & - & - & - & II \\
\hline $\mathrm{H}$ & Eua & $\mathrm{xm}$ & Euphorbia virgata & - & - & - & + & - & - & + & + & - & - & + & - & - & - & + & II \\
\hline $\mathrm{H}$ & Eur & $\mathrm{ms}$ & Ajuga reptans & - & - & $\mathrm{r}$ & - & - & - & - & - & - & - & - & - & - & $\mathrm{r}$ & - & I \\
\hline G & Eua & $\mathrm{ms}$ & Allium oleraceum & - & - & - & - & - & - & - & - & - & - & - & - & $\mathrm{r}$ & - & - & I \\
\hline Th & Eur & $\mathrm{xm}$ & Alyssum alyssoides & - & - & - & - & - & $\mathrm{r}$ & - & - & - & - & - & $\mathrm{r}$ & - & - & - & I \\
\hline $\mathrm{H}$ & Eua & $\mathrm{xm}$ & Artemisia pontica & - & - & - & - & - & - & - & - & - & - & $\mathrm{r}$ & $\mathrm{r}$ & - & - & - & I \\
\hline $\mathrm{H}$ & Eua & $\mathrm{xm}$ & Bromus inermis & - & - & - & - & - & - & + & - & - & - & $\mathrm{r}$ & - & - & - & $\mathrm{r}$ & I \\
\hline Th & Eua & $\mathrm{xm}$ & Buglossoides arvensis & - & - & - & - & - & - & $\mathrm{r}$ & - & - & - & - & - & $\mathrm{r}$ & - & - & I \\
\hline $\mathrm{H}$ & Eua & $\mathrm{ms}$ & Carex polyphylla & - & - & - & - & - & - & - & - & - & - & - & - & - & - & $\mathrm{r}$ & I \\
\hline G & Eua & $\mathrm{xm}$ & Carex praecox & - & - & - & - & + & - & + & - & - & - & - & - & - & - & - & I \\
\hline Th & Adv & $\mathrm{ms}$ & Erigeron annuus & - & - & - & - & - & - & - & - & - & $\mathrm{r}$ & - & - & - & - & $\mathrm{r}$ & I \\
\hline $\mathrm{H}$ & Euc & $\mathrm{xm}$ & Koeleria pyramidata & - & - & - & - & - & - & - & $\mathrm{r}$ & - & - & - & - & - & - & - & I \\
\hline $\mathrm{H}$ & Eua & $\mathrm{ms}$ & Linaria vulgaris & - & - & - & - & - & $\mathrm{r}$ & - & - & - & - & - & - & - & - & + & I \\
\hline
\end{tabular}


Pavel PÎNZARU \& al.

\begin{tabular}{|c|c|c|c|c|c|c|c|c|c|c|c|c|c|c|c|c|c|c|c|}
\hline & & & & & & & & & & & & & & & & \\
\hline $\mathrm{H}$ & Eua & $\mathrm{ms}$ & Phleum phleoides & - & - & - & - & - & - & - & + & - & $\mathrm{r}$ & - & - & - & - & - & I \\
\hline $\mathrm{H}$ & Eua & $\mathrm{xm}$ & Pilosella bauchinii & - & - & - & - & - & $\mathrm{r}$ & - & $\mathrm{r}$ & - & - & - & - & - & - & $\mathrm{r}$ & I \\
\hline $\mathrm{H}$ & Eua & $\mathrm{xm}$ & Poa angustifolia & - & - & - & + & - & - & - & - & - & - & - & - & - & - & - & I \\
\hline $\mathrm{H}$ & Eur & $\mathrm{xm}$ & Polygala comosa & - & - & - & - & + & - & - & - & - & - & - & + & - & + & - & I \\
\hline $\mathrm{H}$ & Eua & $\mathrm{xm}$ & Potentilla argentea & - & - & - & - & - & - & - & - & - & $\mathrm{r}$ & - & - & - & - & - & I \\
\hline $\mathrm{H}$ & Eua & $\mathrm{xm}$ & Poetntilla recta & - & - & - & - & - & + & - & - & - & - & + & - & - & - & - & I \\
\hline $\mathrm{H}$ & Eua & $\mathrm{ms}$ & Tanacetum vulgare & - & - & - & - & - & - & - & - & - & $\mathrm{r}$ & + & - & - & - & - & I \\
\hline Ch & Eua & $\mathrm{xm}$ & $\begin{array}{l}\text { Thymus pannonicus } \\
\text { var. marschallianus }\end{array}$ & - & - & - & - & - & $\mathrm{r}$ & - & $\mathrm{r}$ & - & - & - & - & - & - & - & I \\
\hline $\mathrm{TH}$ & Euc-M & $\mathrm{ms}$ & Tragopogon dubius & - & - & - & - & - & - & $\mathrm{r}$ & - & - & - & - & - & - & $\mathrm{r}$ & - & I \\
\hline Th & Eur & $\mathrm{ms}$ & Valerianella locusta & - & - & - & - & - & - & - & - & $\mathrm{r}$ & - & - & - & - & - & - & I \\
\hline Th & Med & $\mathrm{ms}$ & Trifolium campestre & - & - & - & - & - & - & - & + & - & - & - & - & - & - & $\mathrm{r}$ & I \\
\hline
\end{tabular}

Place and date of the relevés: 1-3, glade no. 1, plot no. 52, Stejăreni village, Strășeni district, 10.VI.2020, 21.VII.2020, 11.IX.2020; 4-12 (*6 -typus), glade no. 2, plot no. 43, Stejăreni village, Strășeni district, 10.VI.2020, 21.VII.2020, 11.IX.2020; 13-15, glade no. 3, polt no. 43, Stejăereni village, Strășeni district, 10.VI.2020, 21.VII.2020, 11.IX.2020. 


\section{Conclusions}

The association Euphorbio valdevillosocarpae-Inuletum salicinae Pînzaru, Cantemir et Jardan ass. nova includes plant communities of hemicryptophytes (74.1\%), mesophiles and xeromesophiles, formed on high hills (330-336 m altitude), on slightly acidic, typical gray soils.

In the floristic composition, the Eurasian elements predominate (53,7 \%), followed by the European (16.6\%) and Central-European ones (6.4\%). The differential species Euphorbia valdevillosocarpa Arvat et Nyár. is a Central European geoelement (endemic), therefore the association can also be considered Central European (Eastern).

The association Euphorbio valdevillosocarpae-Inuletum salicinae Pînzaru, Cantemir et Jardan ass. nova has been included in the alliance Trifolion medii T. Müller 1962, order Origanetalia vulgaris T. Müller 1962, class TRIFOLIO-GERANIETEA SANGUINEI T. Müller 1962.

It has been proposed to include the association Euphorbio valdevillosocarpaeInuletum salicinae in the List of Rare Plant Associations of the Republic of Moldova, with high conservation value.

\section{Notes on contributors}

Pavel PÎNZARU is a professor, PhD, botanist with a special interest in phytocoenology, floristics and systematics. The has published over 195 scientific papers on the flora and vegetation of Bessarabia and Italy. He is co-author on the "Red Book of the Republic of Moldova" $(2001,2015)$, the "Flora of Bessarabia volume” I, II, III (2011, 2016, 2020), is author the "Pteridophytes in the Republic of Moldova” (2019) and the "Rare plants in rocky regions of the Republic of Moldova” (2020).

Valentina CANTEMIR is a doctor of biological sciences, specialist in the field of plant biology, botany, plant systematic, fossil and contemporary flora of the Republic of Moldova and neighboring regions. Author of about 200 scientific papers, including co-author of 10 monographs "Red Book of the Republic of Moldova" ed. 2, 3, (2001, 2015); The vegetal world of Moldova, vol. 2, 3 "Flowering plants" - I, II (2005, 2006); “Flora of Bessarabia” vol. I, II, III (2011, 2016, 2020).

Natalia JARDAN is a botanist, $\mathrm{PhD}$, vice-director of "Codrii”" Reserve with a special interest in floristics, silviculture and conservation of biodiversity. She has published over 30 scientific papers. She is co-author of the "Red Book of the Republic of Moldova" (2015), the "Survey of the biological diversity of the "Codrii” Reserve” (2011).

\section{References}

BENSETTITI F., BOULLET V., CHAVAUDRET-LABORIE C. \& DENIAUD J. (coord.) 2005. Cahiers d'habitats. Natura 2000. Connaissance et gestion des habitats et des espèces d'intérêt communautaire. Tome 4 - Habitats agropastoraux. Volume 2. MEDD/MAAPAR/MNHN. Édit. La Documentation française, Paris, 487 p. + cédérom. Source).

BRAUN-BLANQUET J. 1964. Pflanzensoziologie. Grundzüge der Vegetationskunde. Ed. 3. Wien: Springer Verlag, 855 pp.

CHIFU T. (ed.), IRIMIA I. \& ZAMFIRESCU O. 2014. Diversitatea fitosociologică a vegetaţiei României. II. Vegetaţia erbacee antropizată. Tom. 1. Vegetaţia pajiştilor. Iaşi: Institutul European, 659 pp.

COLDEA G. (ed.), OPREA A., SÂRBU I., SÎRBU C. \& ŞTEFAN N. 2012. Les associations végétales de Roumanie. Tome 2. Les associations anthropogénes. Cluj-Napoca: Presa Universitară Clujeană, 482 pp.

CRISTEA V., GAFTA D. \& PEDROTTI F. 2004. Fitosociologie. Cluj-Napoca: Presa Universitară Clujeană, 394 pp. 


\section{Pavel PÎNZARU \& al.}

DUBYNA D. V., DZIUBA T. P. \& IEMELIANOVA S. M. 2019. Prodrome of the vegetations of Ukraine. Kyiv: Naukova dumka.

GELTMAN D. V. 1996. Euphorbia L. In: TZVELEV N. N. (eds.). Flora Europae Orientalis. T. IX. Petropoli: Mir i Semia-XCV, pp. 262-286.

MUCINA L., BULTMANN H., DIERßEN K. \& al. 2016. Vegetation of Europe: hierarchical floristic classification system of vascular plant, bryophyte, lichen, and algal communities. In: Applied Vegetation Science. 19. Suppl. 1: 3-264.

NEDEALCOV M., RĂILEAN V., CHIRICĂ L. \& al. 2013. Atlas. Resursele climatice ale Republicii Moldova = Atlas Climatic resources of the Republic of Moldova. Chişinău: Î. E. P. Ştiinţa, 76 pp.

PÎNZARU P. \& SÎRBU T. 2016. Flora vasculară din Republica Moldova (Lista speciilor şi ecologia). Ed. 2. Chişinău: Tipografia UST, 261 pp.

SÂRBU I., ŞTEFAN N. \& OPREA A. 2013. Plante vasculare din România. Determinator ilustrat de teren. Bucureşti: Edit. Victor B Victor, 1317 pp.

URSU A. 2011. Solurile Moldovei. Chişinău: Ştiinţa. ISBN 978-9975-67-572-7, 324 pp.

*** 2015. Cartea Roşie a Republicii Moldova $=$ The Red Book of the Republic of Moldova. Ed. 3. Col. Red. DUCA G. \& al. Chişinău: Ştiinţa, Part. I: 11-231.

${ }^{* * *}$ Legea privind fondul ariilor naturale protejate de stat. Nr. 1538 din 25.02.1998. Monitorul Oficial din 16.07.1998, Nr. 066 art. Nr. 442.

\section{How to cite this article:}

PÎNZARU P., CANTEMIR V. \& JARDAN N. 2020. Euphorbio valdevillosocarpae-Inuletum salicinae ass. nova Pînzaru, Cantemir \& Jardan (Trifolion medii T. Müller 1962) in the Republic of Moldova. J. Plant Develop. 27: 175-185. https://doi.org/10.33628/jpd.2020.27.1.175 